\title{
The Finite Moment Log Stable Process and Option Pricing
}

PETER CARR

New York University

LIUREN WU

Fordham University

Follow this and additional works at: https://fordham.bepress.com/crif_working_papers

Part of the Finance and Financial Management Commons

\section{Recommended Citation}

CARR, PETER and WU, LIUREN, "The Finite Moment Log Stable Process and Option Pricing" (2002). CRIF Working Paper series. 19.

https://fordham.bepress.com/crif_working_papers/19 


\title{
The Finite Moment Log Stable Process and Option Pricing
}

\author{
Peter CARR and LiUREN WU*
}

March 25, 2002; first draft: February 21, 2000

* Peter Carr is from the Courant Institute, New York University; 251 Mercer Street, New York, NY 10012; tel: (212) 2603765; pcarrenyc.rr.com. Liuren Wu is from the Graduate School of Business, Fordham University; 113 West 60th Street, New York, NY 10023; tel: (212) 636-6117; wu@fordham.edu. The authors thank Rick Green (the editor), an anonymous referee, Alexander David, Patrick Hagan, Takaki Hayashi, Andrew Matytsin, J. Huston McCulloch, Murad Taqqu, Eric Reiner, and participants of the 2001 WFA conference and the 2000 Columbia Mathematical Finance Seminar for helpful suggestions. 


\title{
The Finite Moment Log Stable Process and Option Pricing
}

\begin{abstract}
We document a surprising pattern in market prices of S\&P 500 index options. When implied volatilities are graphed against a standard measure of moneyness, the implied volatility smirk does not flatten out as maturity increases up to the observable horizon of two years. This behavior contrasts sharply with the implications of many pricing models and with the asymptotic behavior implied by the central limit theorem (CLT). We develop a parsimonious model which deliberately violates the CLT assumptions and thus captures the observed behavior of the volatility smirk over the maturity horizon. Calibration exercises demonstrate its superior performance against several widely used alternatives.
\end{abstract}

JEL Classification Codes: G12, G13, F31, C14.

KEY WORDS: Volatility smirk; central limit theorem; Lévy $\alpha$-stable motion; self-similarity; option pricing. 
Ever since the stock market crash of 1987, the U.S. stock index options market has been exhibiting a consistent pattern documented by academics and practitioners alike. At a given maturity level, the Black and Scholes (1973) implied volatilities for out-of-the-money puts are much higher than those of out-of-the-money calls. ${ }^{1}$ This phenomenon is commonly referred to as the "volatility smirk."

It is, however, less well known that these implied volatilities also exhibit a strong empirical regularity in the maturity direction. ${ }^{2}$ When implied volatilities are graphed against a standard measure of "moneyness", we document that the resulting implied volatility smirk does not flatten out as maturity increases up to the observable horizon of two years. The measure of moneyness for which this observation holds is the logarithm of the strike over the forward, normalized by the square root of maturity.

This maturity pattern of the volatility smirk is somewhat surprising as it ignores the implications of the central limit theorem. It is widely appreciated that the implied volatility smirk is a direct result of conditional non-normality in stock returns. In particular, the downward slope of the smirk reflects asymmetry (negative skewness) in the risk-neutral distribution of the underlying index return, while the positive curvature of the smirk reflects the fat-tails (leptokurtosis) of this distribution. Yet, the central limit theorem implies that under fairly general conditions, the conditional return distribution should converge to normality as the maturity increases. As a result, the volatility smirk should flatten out accordingly.

To account for the volatility smirk at a certain maturity, a large stream of the option pricing literature models the log return as a Lévy process, i.e. a process with stationary independent increments. Prominent examples include the Poisson jump model of Merton (1976), the variance-gamma model of Madan, Carr, and Chang (1998), the log-gamma model of Heston (1993b), and the CGMY model of Carr, Geman, Madan, and Yor (2000). However, for all of these models, the central limit theorem implies that the absolute value of skewness decreases like the reciprocal of the square-root of maturity, while the kurtosis decreases with the reciprocal of maturity (Konikov and Madan (2000)). As a result, the implied volatility smirk obtained from these models flattens out very quickly as maturity increases. Incorporating a persistent stochastic volatility process, e.g. Heston (1993a), slows down the speed of convergence, but does not stop it, so long as the volatility process is stationary. ${ }^{3}$ 
To prevent the flattening of the volatility smirk, we develop a parsimonious option pricing model which deliberately violates the conditions leading to the validity of the central limit theorem. One of the key conditions for the central limit theorem to hold is that the return distribution has finite second moments. In our model, the return distribution of the underlying index has infinite moments for any order of two or greater. As a result, the central limit theorem no longer applies. Nevertheless, our model guarantees that all moments of the index level itself are finite. The finiteness of these price moments guarantees the existence of an equivalent martingale measure and the finiteness of option prices at all maturities.

To combine infinite return moments with finite price moments, we model returns as driven by an $\alpha$-stable motion with maximum negative skewness. The $\alpha$-stable motion is a Lévy process whose departure from Brownian motion is controlled by the tail index $\alpha \in(0,2]$. Setting $\alpha=2$ degenerates the $\alpha$-stable motion into a Brownian motion and our model into the Black-Scholes model. Setting $\alpha$ below two induces a pure jump processes with fat-tails in the return distribution. In contrast to a standard Poisson or compound Poisson process, this pure jump process has an infinite number of jumps over any time interval, allowing it to capture the extreme activity traditionally handled by diffusion processes. Most of the jumps are small and may be regarded as approximating the transition from one decimalized price to another one nearby. We allow $\alpha$ to be a free parameter, whose exact value is determined by calibrating to market option prices.

Like the Brownian motion, the $\alpha$-stable motion exhibits a self-similarity or stability property. This property means that the distribution of the $\alpha$-stable motion over any horizon has the same shape, upon scaling. As a result, with $\alpha<2$, the risk-neutral distribution has fat tails at all horizons. Thus, our model can generate the maturity pattern of the volatility smirk observed in the S\&P index options market. Our specification also has a simple analytical form for the characteristic function of the return. Many standard contingent claims can then be readily priced by the fast Fourier transform (FFT) method of Carr and Madan (1999).

The relevance of $\alpha$-stable motions for option pricing has been recognized previously. For example, Janicki, Popova, Ritchken, and Woyczynski (1997), Popova and Ritchken (1998) and, Hurst, Platen, and Rachev (1999) have worked on option pricing in a symmetric $\alpha$-stable security market. However, modeling log returns by symmetric $\alpha$-stable motions generates infinite price moments, and hence po- 
tentially infinite call values. The key innovation of our paper is the imposition of maximum negative skewness to the $\alpha$-stable Lévy motion. With maximum negative skewness, the process retains the key advantage of the stable law, i.e. fat tails at every maturity. However, in contrast to symmetric stable laws, our asymmetric stable motion also delivers finite conditional moments of all orders for the index level and hence finite call values. Furthermore, the asymmetric nature of our specification also agrees with the observed volatility smirk of index options.

In related work, McCulloch $(1987,1996)$ achieves finite call values by assuming that the marginal utilities of assets follow a logstable process with maximum negative skewness. Although the log return is allowed to have long tails on both sides and the conditional expectation of the stock price is infinite under the objective measure, the marginal utility weighted stock price has only one long tail to the left, and hence has a finite expected value. In contrast, we leave preferences and the process under the objective measure unspecified. Our model incorporates maximum negative skewness directly into the Lévy $\alpha$-stable motion describing the risk-neutral process of the underlying asset. While our specification is supported by the marginal utility interpretation in McCulloch $(1987,1996)$, our focus on the risk-neutral process permits consistency with alternative fundamental considerations, while still serving the useful task of pricing options and other derivative securities.

The stochastic component of our log price process is a pure jump process with no continuous component. At any time, the underlying index can jump to any nonnegative value. This implies that in our model, the risk in an index option position cannot be eliminated via dynamic trading in futures on the underlying index. Although a riskless portfolio can be formed by dynamically trading in a continuum of options, this form of dynamic trading must be regarded as a physical impossibility at present. As a result, the absence of arbitrage implies that there are many possible risk-neutral measures which are equivalent to some assumed statistical measure and consistent with observed asset prices. In determining option prices through time, the market does select some unique process for the prices of options, perhaps by reference to some unspecified equilibrium model. The selection by a modeler of a particular risk-neutral process for the underlying index can be interpreted as a way to indirectly select a particular risk-neutral process and a corresponding initial value for each member of the family of options written on the index. The consistency of this choice with the one made by the market can be measured by capturing the extent to which the model option prices match the market option prices 
across all strikes, maturities, and dates. Thus, we regard the Lévy $\alpha$-stable process which we use to specify the risk-neutral process for index returns as a way to indirectly but exogenously impose a unique arbitrage-free stochastic process and initial value for each member of the option family.

To show that our choice is validated by the time series and cross sectional behavior of observed option prices, we calibrate our model and test it against a number of alternatives using about one year's worth of daily S\&P 500 index option data. The results indicate that (1) despite the parsimony of our model, it has superior explanatory power over all alternatives tested, and (2) the key weakness in traditional Lévy pure jump and jump-diffusion models is their inability to simultaneously fit volatility smirks at different maturities.

Since we only observe prices of options with up to two years to maturity, our documented persistence of the volatility smirk only applies to this period. In principle, the negligible decay over this period can also be captured by combining a highly persistent stochastic volatility process with a jump component, e.g. Bakshi, Cao, and Chen (1997) and Bates (2000). For such processes, the risk-neutral distribution of the asset return would eventually converge to normal, in contrast to the implications of our model. Instead of modifying an existing model to slow down the undesired convergence to normality, our approach is to start from a different process which has stability of the (non-normal) distribution as its defining property. As a result, our model is much more parsimonious in that it has only two free parameters. Nonetheless, the empirical performance is on par with the more complicated models.

This paper is organized as follows. The next section systematically documents the maturity pattern of the volatility smirk for S\&P 500 index options. Section II introduces our specification of the riskneutral index dynamics and illustrates the procedure used to determine option prices. Section III deals with the design and results of our empirical comparisons using S\&P 500 index options. Section IV addresses potential extensions of the our model and the asymptotic properties of the implied volatility smirk. Section V briefly summarizes the paper and suggests some future research. 


\section{Maturity Variation in the Volatility Smirk}

\section{A. Data and Estimation Issues}

To document the maturity variation in the volatility smirk, we have obtained daily closing bid and ask price quotes (and their corresponding implied volatilities) on out-of-the-money S\&P 500 index options across all strikes and maturities from April 6th, 1999 to May 31st, 2000 (290 business days). We also have daily closing futures prices corresponding to each option maturity. Our exclusive use of out-ofthe-money options is an industry convention, arising from their greater liquidity and model sensitivity than their in-the-money counterparts.

We apply the following filters to the data: (1) the time to maturity is greater than five business days; (2) the bid price quote is positive; (3) the ask is no less than the bid. After applying these filters, we also plot the mid implied volatility for each day and maturity against strike prices to visually check for obvious outliers. After removing these outliers, we have 62,950 option quotes left over a period of 290 business days.

\section{B. The Smoothed Implied Volatility Surface}

In Figure 1, we plot the average implied volatility surface across maturity and moneyness, where the latter is defined as:

$$
d=\frac{\ln (K / F)}{\sigma \sqrt{\tau}} .
$$

In this definition, $K$ and $F$ denote the strike price and futures price corresponding to the option term $\tau$, while $\sigma$ denotes some measure of the average volatility of the index. The use of the constant $\sigma$ in the denominator of (1) is an industry convention designed to allow comparison across stocks, and to allow a simple interpretation of this moneyness measure as roughly the number of standard deviations that the log strike is away from the log forward price in the Black-Scholes model. ${ }^{4}$ Furthermore, Backus, Foresi, and $\mathrm{Wu}$ (1997) show that the definition of moneyness used in (1) allows the slope and curvature of the volatility smirk to be transparently related to the skewness and kurtosis of the underlying riskneutral return distribution. In Figure 1, we use $\sigma=27.4 \%$, which is the average over all of the implied 
volatility quotes. We use the mid point of the bid and ask for the implied volatility quotes. The implied volatility $(I V)$ surface as a function of moneyness and maturity is obtained via nonparametric regression with standard independent Gaussian kernels and a default bandwidth selection. ${ }^{5}$

Insert Figure 1 About Here.

Figure 1 indicates that at each maturity, implied volatilities decline almost linearly as moneyness increases. ${ }^{6}$ A striking feature of Figure 1 is the lack of variation in the slope of this line as the maturity increases. This lack of variation contrasts sharply with the flattening observed in the currency options market by Backus, Foresi, and Wu (1997).

Aï-Sahalia and Lo (1998) report a slight flattening in their nonparametrically estimated implied volatility smirk (see their Figure 4 ). However, the horizontal axis of their plot uses $K / F$ for moneyness. This moneyness measure distorts the link between the slope and curvature of the volatility smirk and the skewness and kurtosis of the risk-neutral distribution. Consistent with this observation, their nonparametrically estimated risk-neutral density exhibits skewness and kurtosis which increases with maturity (see their Figure 7), even though their plotted smirk flattens out with maturity.

\section{A Formal Test of the Maturity Pattern}

In this section, we augment our casual observations based on Figure 1 with formal statistical tests. Specifically, for each day, we perform the following two-step regression:

$$
\begin{aligned}
I V_{j} & =a+b_{j} d_{j}+e_{j}, \quad \text { at each maturity } \tau_{j}, \\
b_{j} & =c_{0}+c \tau_{j}+\varepsilon .
\end{aligned}
$$

The first regression estimates the slope of the smirk, $b_{j}$, at each maturity $\tau_{j}$. The second step regresses this estimate of the smirk slope on the maturity and thus captures the maturity pattern of the smirk slope. For the first step, we restrict the moneyness to be in the approximately linear range of $d \in[-2,0]$. We further require at least five data points for each regression. ${ }^{7}$ 
The left panel of Figure 2 depicts the term structure of the smirk slope, $b_{j}$, at each of the 289 business days tested. Visual inspection indicates that (1) the term structure is in most cases nonlinear in maturity and (2) downward sloping term structures dominate upward sloping ones. Bearing in mind that smirk slopes are negative, the downward sloping term structure implies that smirk slopes steepen as maturity increases. Indeed, for 195 out of the 289 days tested, the $t$-statistics for the estimates $\widehat{c}$ are less than -1.96 , while for no days is the $t$-statistic greater than 1.96. In Panel A of Table I, we also report the estimate of a stacked regression on the maturity pattern. The estimate $\widehat{c}$ has a $t$-statistic of -19.32 , which is significantly negative for any reasonable confidence interval. The fit of the stacked regression is depicted by the solid line in the right panel of Figure 2.

Insert Figure 2 About Here.

\section{Insert Table I About Here.}

As an alternative test, in Panel B of Table I, we report the estimates of a stacked one-step multivariate regression: ${ }^{8}$

$$
I V=c_{0}+c_{1} d+c_{2} \tau+c_{3}(\tau d)+e .
$$

While $c_{1}$ captures the slope of the volatility smirk at a fixed maturity and $c_{2}$ captures the term structure of implied volatility at a fixed moneyness, our focus is on the estimate of $c_{3}$, which captures the maturity pattern of the volatility smirk. If the volatility smirk flattens out as maturity increases, we would expect a positive estimate for $c_{3}$. The estimate for $c_{3}$ from the stacked regression is overwhelmingly negative, implying that the implied volatility smirk actually steepens as maturity increases.

Both the smoothed implied volatility surface and the formal regression tests point to a robust feature of the S\&P 500 index options market. When graphed against the appropriate measure of moneyness, the implied volatility smirk does not flatten out as maturity increases. As a result, there is no evidence 
that the risk-neutral density of the index return is converging to normal within the observable maturity range. Hence, the central limit theorem may not be applicable to the market for S\&P 500 index options. Clearly, a new class of models is required in order to account for the behavior of U.S. equity index option prices.

\section{The FMLS Process and Option Pricing}

The empirical results in the previous section indicate that the left tail of the risk-neutral return distribution remains "fat" as maturity increases. Traditionally, this has been interpreted as the result of a "slow" convergence to normality. Persistent stochastic volatility processes have been proposed to generate the slow convergence. Our modeling effort starts from a different perspective. We regard the data as evidence of a potential violation of the conditions supporting the central limit theorem and search for processes under which the risk-neutral distribution does not converge to normality at all as maturity increases.

In selecting from classes of stochastic processes, an obvious candidate is the $\alpha$-stable class, under which the thickness of the tails of the distribution are invariant to time aggregation. The parameter $\alpha$ governs the thickness of the tails relative to the central mass. The Brownian motion applied in the Black-Scholes model is a very special example of the $\alpha$-stable class with $\alpha=2$. To generate the observed fat tails in the return distribution, we need $\alpha<2$. Unfortunately, this specification implies infinite variance for the log return and hence raises concerns on the existence of a martingale measure and the finiteness of option values.

To deal with this issue, we focus attention on a second parameter $\beta \in[-1,1]$ which governs the degree of skewness in the distribution. Our key innovation is to restrict attention to the case of $\beta=-1$, which forces maximum negative skewness into the Lévy $\alpha$-stable motion. When $\beta=-1$, we prove that the conditional moments of all orders exist for the index level, even though the log return continues to have infinite variance. To distinguish our process from other members of the stable class, we christen the process governing index levels as the Finite Moment Log Stable (FMLS) process. Although our intent in this parametric restriction is to produce finite option prices, our specification has the added 
attraction of capturing the highly skewed feature of the implied density for log returns, a feature that cannot be captured by either a Brownian motion or a symmetric Lévy $\alpha$-stable motion.

To formally describe the FMLS process, first let $L_{t}^{\alpha, \beta}$ denote a standardized Lévy $\alpha$-stable motion with tail index $\alpha \in(0,2]$ and skew parameter $\beta \in[-1,1]{ }^{9}$ As the $\alpha$-stable motion is standardized, it is a martingale and hence is a candidate as the driver of the risk-neutral process for discounted index levels. As the $\alpha$-stable motion is a Lévy process, it has independent and stationary increments. The increment $d L_{t}^{\alpha, \beta}$ has an $\alpha$-stable distribution with zero drift, dispersion of $d t^{1 / \alpha}$, and a skew parameter $\beta: L_{\alpha}\left(0, d t^{1 / \alpha}, \beta\right)$.

Next, let $S_{t}$ denote the spot level of the index at time $t \in[0, \mathcal{T}]$, where $\mathcal{T}$ is some arbitrarily distant horizon. We assume that $S_{t}$ is realized as coordinate process on the canonical space $\Omega=\mathbb{R}^{+}$with its natural filtration $\left(\mathcal{F}_{t}\right)_{t \geq 0}$ and $\mathcal{F}=\vee_{t \in \mathbb{R}_{+}} \mathcal{F}_{t}$. We further assume that the risk-neutral probability measure $\mathbb{Q}$ is such that the index level obeys the following stochastic differential equation:

$$
d S_{t} / S_{t}=(r-q) d t+\sigma d L_{t}^{\alpha,-1}, \quad t \in[0, \mathcal{T}], \alpha \in(1,2), \sigma>0
$$

where $r$ and $q$ denote respectively the continuously compounded risk free rate and dividend yield, both of which are assumed to be deterministic. In our specification for the index dynamics, we set $\beta=-1$ to achieve finite moments for index levels and negative skewness in the return density. We also restrict $\alpha \in(1,2)$ so that the return has the support of the whole real line. When $\alpha \leq 1$ and $\beta=-1$, the $\alpha$-stable motion $L_{t}^{\alpha,-1}$ has all of its support on the negative half of the real line. As a result, the index level would become bounded above.

The process in (3) is specified directly under the risk-neutral measure $\mathbb{Q}$. This specification is consistent with our focus on the options data and on the risk-neutral distribution of the log return. We refer to the marginal utility specification in McCulloch $(1987,1996)$ as one way to reconcile the time series properties of the log return with the risk-neutral properties implied by option prices.

Under the FMLS model, the innovation in the log return is an $\alpha$-stable random variable with $\alpha \in$ $(1,2]$. We now summarize the relevant properties of this distribution. 
Property 1 Let $x \sim L_{\alpha}(\theta, \sigma, \beta)$ denote an $\alpha$-stable random variable with $\alpha \in(1,2], \theta \in \Re, \sigma \geq 0$, and $\beta \in[-1,1]$.

1. The characteristic function of $x$ is given by:

$$
\phi_{x}(u) \equiv \mathbb{E} e^{i u x}=\exp \left[i u \theta-|u|^{\alpha} \sigma^{\alpha}\left(1-i \beta(\operatorname{sgn} u) \tan \frac{\pi \alpha}{2}\right)\right], \quad u \in \mathbb{R} .
$$

$\beta$ is irrelevant when $\alpha=2$ (Samorodnitsky and Taqqu 1994, page 5).

2. When $\alpha<2$, the tails are "fat" and the tail probabilities behave like $\lambda^{-\alpha}$.

$$
\lim _{\lambda \rightarrow \infty} \lambda^{\alpha} P(x>\lambda)=C_{\alpha} \frac{1+\beta}{2} \sigma^{\alpha}, \quad \lim _{\lambda \rightarrow \infty} \lambda^{\alpha} P(x<-\lambda)=C_{\alpha} \frac{1-\beta}{2} \sigma^{\alpha}
$$

for some parameter $C_{\alpha}$. The only exception is if $|\beta|=1$. When $\beta=-1$, the right tail is a "thin" tail decaying faster than $\lambda^{-\alpha}$. As $\lambda \rightarrow \infty$,

$$
P(x>\lambda) \sim \frac{1}{\sqrt{2 \pi \alpha(\alpha-1)}}\left(\frac{\lambda}{\alpha \hat{\sigma}_{\alpha}}\right)^{-\alpha /(2(\alpha-1))} \exp \left(-(\alpha-1)\left(\frac{\lambda}{\alpha \hat{\sigma}_{\alpha}}\right)^{\alpha /(\alpha-1)}\right)
$$

for some parameter $\widehat{\sigma}_{\alpha}$ (Zolotarev 1986, Theorem 2.5.3).

3. The two-sided Laplace transform of $x$ is not finite unless $\beta=1$. When $\beta=1$, the Laplace transform is given by:

$$
\mathcal{L}_{x}(\lambda) \equiv \mathbb{E}\left[e^{-\lambda x}\right]=\exp \left(-\lambda \theta-\lambda^{\alpha} \sigma^{\alpha} \sec \frac{\alpha \pi}{2}\right), \quad \Re \lambda \geq 0
$$

(Samorodnitsky and Taqqu, 1994, page 15, Proposition 1.2.12).

4. For any $0<\alpha<2$,

$$
X \sim L_{\alpha}(0, \sigma, \beta) \Leftrightarrow-X \sim L_{\alpha}(0, \sigma,-\beta)
$$

(Samorodnitsky and Taqqu, 1994, page 11, Property 1.2.4).

5. For any $0<\alpha<2, \mathbb{E}|x|^{p}<\infty$ for any $0<p<\alpha$ and $\mathbb{E}|x|^{p}=\infty$ for any $p>\alpha$. (Samorodnitsky and Taqqu, 1994, page 18, Property 1.2.16). 
In the literature, an $\alpha$-stable random variable is often defined directly by its characteristic function (Property 1.1). While the tails in a normal distribution decay exponentially, an $\alpha$-stable tail with $\alpha<2$ decays according to a power law, as described in (5). It is thus "fatter" than a normal tail. In the extreme case of $\beta=-1$, only the left tail is fat. The right tail is thin and decays exponentially. The opposite is true with $\beta=1$. Because of this, the two-sided Laplace transform of the density is finite only when $\beta=1$. Property 1.4 states that $X$ is the mirror image of $-X$ under the stable law. Property 1.5 states that the moments of an $\alpha$-stable random variable with $\alpha<2$ are finite only when the order of the moment is lower than $\alpha$.

Based on the above properties of an $\alpha$-stable random variable, we prove the following proposition for our FMLS model.

\section{Proposition 1 Under the FMLS model specified in (3)}

1. The log return $s_{\tau}=\ln S_{T} / S_{t}$ over horizon $\tau=T-t$ has an $\alpha$-stable distribution: $s_{\tau} \sim L_{\alpha}((r-$ $\left.q+\mu) \tau, \sigma \tau^{1 / \alpha},-1\right)$, with $\mu=\sigma^{\alpha} \sec \pi \alpha / 2$. In particular, the variance of the log return $s_{\tau}$, or any moments of order higher than $\alpha$, is not finite.

2. The $n$-th conditional moment of the spot index level $S_{T}$, for all $n \geq 0$, is well-defined and is given by

$$
\mathbb{E}_{t}\left[S_{T}^{n}\right]=S_{t}^{n} \exp \left(n(r-q+\mu) \tau-\tau(n \sigma)^{\alpha} \sec \frac{\pi \alpha}{2}\right)<\infty, \forall n \in[0, \infty),
$$

where $\mathbb{E}_{t}[\cdot]$ denotes expectation under measure $\mathbb{Q}$ conditional on the filtration $\mathcal{F}_{t}$.

The proposition formalizes the key features of the FMLS model. Infinite variance in log returns is essential in escaping the purview of the central limit theorem (CLT). On the other hand, finite moments in spot index levels are needed for the existence of a martingale measure and finite option values.

Since an exponential map will be applied to log returns in order to generate prices, finite price moments can be achieved in the $\alpha$-stable context by changing the default setting of the skew parameter from zero to its negative extreme: $\beta=-1$. Under this specification, the left tail of the log return remains "fat", decaying according to a power law. However, now the right tail is thin and decays exponentially (Property 1.2). The fat left tail continues to generate infinite variance for the log return, and hence the CLT remains irrelevant. However, now the right tail is thin enough so that when we map log returns to 
prices by an exponential function, the price moments are all finite. We need not worry about infinite price variance being generated from the left tail, since price realizations are effectively stopped at zero.

Proof. From (3), by the Lévy property of the $\alpha$-stable motion, we can write the spot index level $S_{T}$ in an exponential form,

$$
S_{T}=S_{t} e^{(r-q) \tau} e^{\mu \tau+\sigma L_{\tau}^{\alpha,-1}}
$$

where $\tau=T-t$ represents the horizon and $\mu$ is a convexity adjustment, made so that the expectation of the last term

$$
\mathbb{E}\left[e^{\mu \tau+\sigma L_{\tau}^{\alpha,-1}}\right]=1
$$

satisfies the martingale condition under the risk neutral measure. Applying Property 1.4 and then Property 1.3 on the Laplace transform, we have

$$
\mathbb{E}\left[e^{\sigma L_{\tau}^{\alpha,-1}}\right]=\mathbb{E}\left[e^{-\sigma L_{\tau}^{\alpha, 1}}\right]=e^{-\tau \sigma^{\alpha} \sec \frac{\pi \alpha}{2}}
$$

The convexity adjustment term is thus given by

$$
\mu=\sigma^{\alpha} \sec \frac{\pi \alpha}{2}<\infty
$$

Note that since the expectation in (10) is finite only when $\beta=-1$, the martingale condition in (9) can only be satisfied under our FMLS specification of $\beta=-1$.

We now rewrite the spot index level as

$$
S_{T}=S_{t} e^{(r-q+\mu) \tau} e^{\sigma L_{\tau}^{\alpha,-1}}
$$

with $\mu$ given in (11). The $\log$ return $s_{\tau} \equiv \ln S_{T} / S_{t}$ is then given by,

$$
s_{\tau} \equiv \ln S_{T} / S_{t}=(r-q+\mu) \tau+\sigma L_{\tau}^{\alpha,-1}
$$

which is obviously $\alpha$-stable distributed with mean $(r-q+\mu) \tau$, dispersion $\sigma \tau^{1 / \alpha}$, tail index $\alpha$, and skewness $\beta=-1$. By Property 1.5, any moment of order higher than $\alpha$ is not finite for such a random variable. 
From (12), we can also compute the $n$th conditional moment of $S_{T}$, for $n \geq 0$,

$$
\mathbb{E}_{t}\left[S_{T}^{n}\right]=S_{t}^{n} e^{n(r-q+\mu) \tau} \mathbb{E}\left[e^{n \sigma L_{t}^{\alpha,-1}}\right]=S_{t}^{n} e^{n(r-q+\mu) \tau-\tau(n \sigma)^{\alpha} \sec \frac{\pi \alpha}{2}}<\infty
$$

The last equality is again obtained from Property 1.3 on Laplace transform.

While $\alpha$-stable distribution has been applied to stock price modeling since the pioneering work of Mandelbrot (1963) and Fama (1965), we are the first to focus on the applicability of the $\alpha$-stable motion with maximum negative skewness in modeling index returns. The confinement to maximum negative skewness is important as it leads to the only $\alpha$-stable specification that generates infinite variance (and higher moments) for the index return, but finite moments of all orders for the index level. Such a property is essential in guaranteeing finite option prices. ${ }^{10}$

To see how the tail index $\alpha$ affects the $n$th moments of the spot index level, we re-arrange equation (8) as

$$
\mathbb{E}_{t}\left[S_{T}^{n}\right]=S_{t}^{n} e^{n(r-q) \tau} \exp \left[\tau \sigma^{\alpha} \sec \frac{\pi \alpha}{2}\left(n-n^{\alpha}\right)\right]
$$

Under a fixed maturity $\tau$, for any $n>1$ and $\sigma \in(0,1)$, the $n$th conditional moment of the spot index level decreases with $\alpha$ and reaches its minimum when $\alpha$ reaches its upper bound of 2 . The conditional mean (first moment, $n=1$ ) of the spot index level is determined by no-arbitrage and does not depend on the magnitude of the tail index $\alpha$. Negative moments of the spot index level $(n<0)$ are infinite.

By setting $\alpha=2$, we obtain the Black-Scholes model as a degenerate case of our model. At $\alpha=2$, the Laplace transform defined in (7) becomes $\mathcal{L}_{x}(\lambda)=\exp \left(\lambda^{2} \sigma^{2}\right)$. Thus, the $\alpha$-stable motion $L_{t}^{2, \beta}$ is related to a standard Brownian motion $W_{t}$ by $L_{t}^{2, \beta}=\sqrt{2} W_{t}$. As indicated in Property 1.1 , the skew parameter $\beta$ is irrelevant at $\alpha=2$.

To obtain theoretical option prices when $\alpha<2$, we note that as the $\log$ return $s_{\tau}$ has an $\alpha$-stable distribution, its characteristic function is given by (4). Under the special restriction of $\beta=-1$, we can also write it in a form analogous to the Laplace transform in (7):

$$
\phi_{s}(u) \equiv \mathbb{E}_{t}\left[e^{i u s_{\tau}}\right]=\exp \left(i u(r-q+\mu) \tau-\tau(i u \sigma)^{\alpha} \sec \frac{\pi \alpha}{2}\right), \quad 1<\alpha<2, \mathfrak{I}(u) \leq 0 .
$$


From Property 1.3 governing the Laplace transform, the restriction $\mathfrak{I}(u) \leq 0$ is needed to make this generalized characteristic function well-defined. Given the generalized characteristic function, the fast Fourier transform (FFT) method of Carr and Madan (1999) can be applied to simultaneously value European calls at a whole spectrum of strike prices. ${ }^{11}$

Our FMLS model has only two free parameters $\sigma$ and $\alpha$, but it can capture the consistent shapes of the volatility smirk exhibited in the S\&P 500 options market. As in the Black-Scholes model, the parameter $\sigma$ controls the width of the risk-neutral distribution and hence the height of the implied volatility smirk. Our additional parameter $\alpha$ controls the extent of the negative slope in the implied volatility smirk. The left panel of Figure 3 illustrates the wide range of implied volatility smirks that can be generated by our FMLS model with different tail index values. The flat line (dotted) is generated by setting $\alpha=2$, which is the Black-Scholes model. As we reduce the magnitude of the tail index $\alpha$, the slope of the implied volatility smirk becomes progressively steeper. As $\alpha$ goes from 2.0 to $1.8,1.5$, and 1.2 , the slope estimate of the implied volatility smirk goes from zero to $-6.71,-10.64$, and $-13.60 .{ }^{12}$ During the 289 business days of our data sample, the steepest smirk has a slope estimate of -9.30 . The flattest smirk has a slope estimate of -1.10 (Refer to Figure 2). Thus, our FMLS model, although extremely parsimonious, can readily generate implied volatility smirks of all observable slopes.

Insert Figure 3 About Here.

Our motivation for working with stable processes is that the thickness of the tails in the return distribution is invariant to time aggregation. This invariance causes the implied volatility smirk to retain its basic shape as maturity increases, as depicted in the right panel of the Figure 3 . There is a slight flattening of the slope in the implied volatility smirk, but it is shown in Appendix B that this effect occurs asymptotically in all models with the reasonable property that implied volatility is asymptotically finite. Thus, the slope flattening depicted in the right panel need not necessarily indicate the beginnings of some asymptotic convergence to normality, but may merely be a consequence of the widely adopted use of the implied volatility slope as the measure of asymmetry in the return distribution. 
In the next section, we show that the stability of the return distribution in the FMLS model distinguishes it from traditional Lévy jump-diffusion models and makes it comparable in performance to much more complicated jump-diffusion/stochastic volatility models.

\section{Model Calibration}

In this section, we investigate the empirical performance of our FMLS model in fitting the typical behavior of S\&P 500 index options. We compare the performance of our two parameter model with other popular option pricing models of increasing complexity. In particular, comparisons are made with the three-parameter variance-gamma (VG) model of Madan, Carr, and Chang (1998) and the fourparameter jump-diffusion (MJD) model of Merton (1976). We also compare with the MJD model with stochastic volatility (MJD-SV), as in Bates (1996) and Bakshi, Cao, and Chen (1997). This last model has seven parameters plus an additional state variable (the current level of instantaneous variance).

Under the VG model, the log return also follows a pure jump Lévy process. The pure jump process is obtained by subordinating an arithmetic Brownian motion by an independent gamma process. Under a risk-neutral measure $\mathbb{Q}$, the characteristic function of the $\log$ return $s_{\tau} \equiv \ln S_{t+\tau} / S_{t}$ is given by

$$
\phi_{s}(u)=e^{i u(r-q+\mu) \tau}\left[1-\left(i u \omega-\frac{1}{2} \sigma^{2} u^{2}\right) \alpha\right]^{-\frac{\tau}{\alpha}},
$$

where $\alpha$ is the variance rate of the gamma process, and $\omega$ and $\sigma^{2}$ are respectively the instantaneous drift and variance of the original Brownian motion. The entity $\mu$ is a convexity adjustment determined by the three free parameters:

$$
\mu=\frac{1}{\alpha} \ln \left[1-\left(\omega+\frac{1}{2} \sigma^{2}\right) \alpha\right]
$$

Under the MJD model, the log return has both a diffusion component and a compound Poisson jump component, where the jump size is assumed to be drawn from a normal distribution. Under a risk-neutral measure $\mathbb{Q}$, the characteristic function of $s_{\tau}$ is given by

$$
\phi_{s}(u)=\exp \left[i u(r-q+\mu) \tau-\frac{1}{2} u^{2} \sigma^{2} \tau+\alpha\left(e^{i u \omega-\frac{1}{2} u^{2} \eta^{2}}-1\right) \tau\right]
$$


where $\sigma^{2}$ denotes the instantaneous variance of the diffusion component, $\alpha$ denotes the mean jump intensity, and $\left(\omega, \eta^{2}\right)$ denotes the mean and variance of the normally-distributed jump size. The convexity adjustment is given by

$$
\mu=-\alpha\left(e^{\omega+\eta^{2} / 2}-1\right)-\frac{1}{2} \sigma^{2}
$$

The MJD-SV model allows the instantaneous variance $v_{t}=\sigma^{2}$ in the MJD model to be stochastic as in Heston (1993a). Under measure $\mathbb{Q}$, the instantaneous variance follows a mean reverting square root process

$$
d v_{t}=\kappa\left(\theta-v_{t}\right) d t+\sigma_{v} \sqrt{v_{t}} d Z_{t}
$$

where $Z_{t}$ is another Brownian motion, possibly correlated with the Brownian motion $W_{t}$ in the return process: $\rho d t=\mathbb{E}\left[d W_{t} d Z_{t}\right]$. The characteristic function of $s_{\tau}$ is

$$
\phi_{s}(u)=\exp \left[i u(r-q+\mu) \tau+\alpha\left(e^{i u \omega-\frac{1}{2} u^{2} \eta^{2}}-1\right) \tau-b(\tau) v_{t}-c(\tau)\right],
$$

where

$$
\begin{aligned}
b(\tau) & =\frac{2 \lambda\left(1-e^{-\eta \tau}\right)}{2 \eta-\left(\eta-\kappa^{*}\right)\left(1-e^{-\eta \tau}\right)} \\
c(\tau) & =\frac{\kappa \theta}{\sigma_{v}^{2}}\left[2 \ln \left(1-\frac{\eta-\kappa^{*}}{2 \eta}\left(1-e^{-\eta \tau}\right)\right)+\left(\eta-\kappa^{*}\right) \tau\right]
\end{aligned}
$$

with

$$
\eta=\sqrt{\left(\kappa^{*}\right)^{2}+2 \sigma_{v}^{2} \lambda}, \quad \kappa^{*}=\kappa-i u \sigma_{v} \rho, \quad \lambda=\left(i u+u^{2}\right) / 2 .
$$

Given the characteristic functions (14) to (17) for the four candidate models, option prices can be computed by the FFT algorithm of Carr and Madan (1999).

We compare the performance of the four models in matching the option price behaviors across both strikes and maturities at each day. Daily calibration is an industry standard and is also widely adopted in academia, e.g. Bakshi, Cao, and Chen (1997). Specifically, at each day, we estimate the vector of model parameters, $\Theta,{ }^{13}$ to minimize the sum of squared pricing errors (SSE) of options across all strikes and maturities,

$$
\operatorname{SSE}(\mathrm{t})=\min _{\Theta} \sum_{i=1}^{N}\left|O_{i}-\hat{O}_{i}(\Theta)\right|^{2}
$$


where $N$ denotes the number of options at date $t, O_{i}$ denotes the observed market price of an option at a certain strike $K$ price and maturity $\tau$, and $\hat{O}_{i}(\Theta)$ denotes the model-implied counterpart of $O_{i}$ as a function of the parameter vector $\Theta$. We repeat the above procedure every day and obtain a time series of parameter estimates and SSE's.

The construction of the pricing error and weighting matrix is a delicate but important issue. For example, the pricing error can be defined on implied volatility, call option price, or put option price. It can be defined as the difference in levels, in log of the levels, or in percentages. In theory, when coupled with an appropriate choices of a weighting matrix, all of these definitions of pricing errors are equivalent. Yet, in practice, a careful choice is imperative in obtaining robust sample estimates. Here, we define the pricing error on call options when $K>F$ and put options when $K \leq F$. Hence, we choose out-of-the-money options across all strike prices. The choice of out-of-the-money options over their in-the-money counterparts at all strike prices in model estimation has become an industry standard. Such a standard arises for several reasons. First, since near-the-money options are more expensive than far out-of-the-money options of the same maturity, our optimization criterion attaches more weight to the more liquid near-the-money options than to the far out-of-the-money ones. Second, in-the-money options have positive intrinsic value which is insensitive to model specification and yet can be the dominant component of the total value. Third, when there is a discrepancy between the market quotes on the out-of-the-money options versus their in-the-money counterparts, ${ }^{14}$ the quotes on out-of-the-money options are in general more reliable, as they are more liquid. The greater liquidity is probably because out-of-the-money options represent a cheaper way to speculate on or hedge against changes in future volatility in the presence of leverage constraints.

For the calibration, we include options of all available maturities greater than five business days. The FFT option pricing algorithm only returns option prices at discrete moneyness levels $k \equiv \ln K / S$ of constant spacing $\Delta k$. Visual inspection indicates that at each date and maturity, the quotes are so close to each other along this moneyness measure that interpolation can be done with little error. As a result, we linearly interpolate to obtain option prices at all moneyness levels within a pre-set range. ${ }^{15}$ We set this range to be between $k \equiv \ln K / S_{t}=-0.3988$ and $k=0.1841$, with a fixed interval of $\Delta k=0.03068$ (a maximum of 20 strike points at each maturity). For the interpolation to work with sufficient precision, we require that at each day and maturity, we have at least five option quotes. We also refrain from 
extrapolating. The number of data points in each of the 289 active business days ranges from 92 to 144 , with an average of 118 sample points per day. In total, we have 34,361 sample data points used in the calibration.

Some of the model parameters have natural constraints on their admissible domains. For example, the tail index $\alpha$ in the FMLS model is constrained to be in the interval $(1,2)$, the volatility parameter $\sigma$ needs to be positive, etc. In our estimation, we transform model parameters to extend their admissible domain to the whole real line and then perform unconstrained optimization using standard numerical procedures.

To compare the performance of different models, we construct a test for non-nested models. Assuming that the pricing errors are iid normally distributed with variance $\sigma^{2}$, the log density function implied by model $k$ is given by

$$
f_{k}\left(e_{i} ; \Theta_{k}\right)=-\frac{1}{2} \ln \left(2 \pi \sigma^{2}\right)-\frac{e_{i}^{2}}{2 \sigma^{2}}
$$

where $e_{i}$ denotes the pricing error on the $i$ th option, and $\Theta_{k}$ is the parameter vector of the model $k$. Since the maximum likelihood estimate for $\sigma^{2}$ is simply the mean squared pricing error: $\mathrm{mse}=\mathbf{e}^{\top} \mathbf{e} / N$, the log likelihood function is given by

$$
\mathcal{L}_{k}\left(\mathbf{e} ; \Theta_{k}\right)=\sum_{i=1}^{N} f_{k}\left(e_{i} ; \Theta_{k}\right)=-\frac{N}{2}\left[1+\ln (2 \pi)+\ln \left(\frac{\mathbf{e}^{\top} \mathbf{e}}{N}\right)\right] .
$$

Under such assumptions, minimizing the sum of squared pricing errors in (19) is equivalent to maximizing the log likelihood function in (20). The parameter estimates can therefore be regarded as maximum likelihood estimates. Furthermore, the log likelihood ratio between the two models $(i$ and $j)$ is given by

$$
L R\left(\Theta_{i}, \Theta_{j}\right)=\mathcal{L}_{i}\left(\mathbf{e} ; \Theta_{i}\right)-\mathcal{L}_{j}\left(\mathbf{e} ; \Theta_{j}\right)
$$

Vuong (1989) constructs a test statistic based on the log likelihood ratio:

$$
\hat{M}_{0}=N^{-1 / 2} L R\left(\Theta_{i}, \Theta_{j}\right) / \hat{\omega}
$$


with $\hat{\omega}^{2}$ being the variance estimate of $\left(f_{i}-f_{j}\right)$. Vuong (1989) proves that $\hat{M}_{0}$ is asymptotically normally distributed $N(0,1)$ under the null that the two models are equivalent in terms of likelihood:

$$
H_{0}: \mathbb{E}\left[f_{i}-f_{j}\right]=0
$$

We hence can form a test on whether the FMLS model is better or worse than the other models by letting model $i$ be the FMLS model and letting $j$ be the VG, MJD, and MJD-SV model. The statistic $\hat{M}_{0}$ is not adjusted for differences in number of free parameters in each model. Adjusted tests can be constructed by adjusting the log likelihood ratio by, for example,

$$
L R_{1}=L R-(p-q) ; \quad \text { or } \quad L R_{2}=L R-\frac{1}{2}(p-q) \ln N
$$

where $p$ and $q$ are the number of parameters in models $i$ and $j$, respectively. The resulting statistics $\hat{M}_{1}$ and $\hat{M}_{2}$ correspond to, respectively, the Akaike (1973) and the Schwartz (1978) information criteria. Their asymptotic distributions are the same as $\hat{M}_{0}$.

Table II reports the sample average and standard deviation of the parameter estimates. We also compute the mean squared pricing error (mse) and the likelihood ratio tests between the FMLS model and the other three models at each day. The sample average and standard deviation of mse and the three tests, $\hat{M}_{j}, j=0,1,2$, are also reported in Table II. Among the three Lévy type models (FMLS, VG, and MJD), our FMLS model not only has the least number of parameters, but it also has the smallest mean squared pricing error. As a result, all three tests indicate that the FMLS model significantly outperforms the VG model and MJD model. The MJD-SV model seems to perform marginally better than our FMLS model in terms of the daily average of the mean squared pricing error. However, the better performance is achieved with five additional free parameters and one additional state variable. ${ }^{16}$ As a result, while the unadjusted test implies that neither model significantly outperforms the other, ${ }^{17}$ the two adjusted test statistics significantly favors our FMLS model for its extreme parsimony.

Insert Table II About Here. 
Insert Figure 4 About Here.

In Figure 4, we adopt the mean parameter estimates in Table II and compute the volatility smirks at different maturities implied by the four models. As expected, the Achilles heel of the VG (bottom left) and the MJD model (bottom right) is that, for a given set of parameters, they cannot simultaneously fit the implied volatility smirk at different maturities. The same weakness holds for all Lévy processes with finite (but nonzero) skewness and kurtosis for log returns, as the absolute values of these moments decrease with maturity by virtue of the central limit theorem. Correspondingly, the volatility smirk flattens out quickly as maturity increases. The MJD-SV model slows down the convergence to normality by incorporating a persistent volatility process. As a result, it also performs well in the maturity dimension (top right). The choice between the two best models tested depends on the criteria to be employed. Parsimony favors our FMLS model, but the MJD-SV model is preferable if one believes that the risk-neutral distribution will start tending to normality beyond the two year horizon.

\section{Extensions and Discussions}

Notwithstanding the favorable results of our comparisons, we do not regard the FMLS model as the final answer to the difficult problem of modelling option prices. After all, log returns under the FMLS model follow a simple pure jump process with iid Lévy increments. One naturally wonders about the merit of an additional diffusion component, as in the jump-diffusion specification of Merton (1976). Furthermore, such a Lévy specification does not capture the well-documented evidence on time-varying and serially dependent volatilities, nor does it capture the time-varying feature of the term structure of the smirk slope, as depicted in the left panel of Figure 2. As such, we regard the FMLS model as a springboard for further extensions which capture this and other finer properties of the options market. In what follows, we briefly discuss some of these potential extensions and their potential merits in practice. We also elaborate on the asymptotic behavior of the implied volatility smirk and its relation, if any, to the asymptotic normality of the risk-neutral return distribution. 


\section{A. Pure Jump Versus Jump Diffusion}

While the FMLS model is a pure jump model, a diffusion component can be added to the model in a very straightforward manner:

$$
d S_{t} / S_{t}=(r-q) d t+\sigma_{1} d L_{t}^{\alpha,-1}+\sigma_{2} d L_{t}^{2}
$$

where $L_{t}^{2}$ is a Brownian motion with variance $2 t$ and is a degenerate case of an $\alpha$-stable motion with $\alpha=2$. The characteristic function of the $\log$ return, $s_{\tau} \equiv \ln S_{t+\tau} / S_{t}$, can be obtained as a direct extension of (14):

$$
\phi_{s}(u)=\exp \left(i u\left(r-q+\sigma_{1}^{\alpha} \sec \frac{\pi \alpha}{2}-\sigma_{2}^{2}\right) \tau-\tau\left(i u \sigma_{1}\right)^{\alpha} \sec \frac{\pi \alpha}{2}-\tau\left(u \sigma_{2}\right)^{2}\right) .
$$

Option pricing is therefore also straightforward.

Our preliminary calibration exercises indicate that the addition of a diffusion component to the FMLS process adds little to the explanatory power. This finding coincides with the recent empirical evidence of Carr, Geman, Madan, and Yor (2000), who also find that the explanatory power of their pure jump CGMY process is not enhanced by the addition of a diffusion component. However, this result regarding the redundancy of a diffusion component is not supported by empirical results from traditional Poisson jump-diffusion models, such as Merton (1976) (MJD). Indeed, in our calibration of the MJD model in Table II, diffusion is a significant component accounting for $5.5 \%$ of the variation. ${ }^{18}$

The varying results regarding the relevance of a diffusion component can be explained by the fine structure of the jump specification in these models. The compound Poisson jump process used in the MJD model has a finite number of jumps over any finite time interval. Thus, the addition of a diffusion process helps in capturing the frequent small moves experienced by most prices. In contrast, the FMLS and CGMY processes experience an infinite number of jumps over any finite time interval, and as a result mimic the infinite variation inherent in all diffusion processes.

To investigate whether there is a jump component and/or diffusion component in the price process of the stock index, Carr and $\mathrm{Wu}$ (2001) propose a simple robust test based upon the asymptotic behavior of options prices as time to maturity approaches zero. They find strong evidence on the presence of a jump component. The evidence on the diffusion component, however, is less conclusive. 


\section{B. Stochastic "Volatility"}

It has been well-documented that while stock returns are approximately uncorrelated, their volatility exhibits strong serial dependence, e.g., Ding, Engle, and Granger (1993) and Ding and Granger (1996). Furthermore, as shown in the left panel of Figure 2, the term structure of the implied volatility also varies daily, suggesting that "volatility" is stochastic under the risk-neutral measure. Since Lévy processes display independent increments, none of the Lévy type models (including our FMLS model) can capture this feature. Traditional jump-diffusion models have been extended to incorporate stochastic volatility, such as the MJD-SV model. At first glance, incorporating stochastic volatility into our FMLS framework appears difficult, since the $\log$ price relatives have infinite variance. Nevertheless, the dispersion parameter $\sigma$ governs the width of the risk-neutral distribution, and it can be randomized while still permitting tractable option pricing models.

Specifically, suppose we apply the Heston (1993a) stochastic volatility process to model the $\alpha$ dispersion: $v_{t}=\sigma^{\alpha}$ :

$$
\begin{aligned}
d S_{t} / S_{t} & =(r-q) d t+v_{t}^{1 / \alpha} d L_{t}^{\alpha,-1} \\
d v_{t} & =\kappa\left(\theta-v_{t}\right) d t+\sigma_{v} \sqrt{v_{t}} d Z_{t} .
\end{aligned}
$$

Using the stochastic time change technique elaborated in Carr and $\mathrm{Wu}$ (2000), we readily derive the characteristic function of the $\log$ return $s_{\tau}$ :

$$
\phi_{s}(u)=\exp \left[i u(r-q) \tau-b(\tau) v_{t}-c(\tau)\right]
$$

with $[b(\tau), c(\tau)]$ having the same form as in (18), only with the following replacements:

$$
\lambda=\left[(i u)^{\alpha}-i u\right] \sec \frac{\pi \alpha}{2}, \quad \kappa^{*}=\kappa .
$$

Since the characteristic function is known, option prices can be computed readily. The empirical performance of this model and related extensions is a subject for future research. 


\section{Volatility Smirk Behavior of Index Options}

The maturity pattern of the implied volatility smirk for S\&P 500 index options is unusual and is not shared by, for example, currency options. For currency options, the implied volatility plot against moneyness looks more like a symmetric smile than a skewed smirk. Furthermore, as maturity increases, the smile readily flattens out rather than retaining its shape as in the S\&P 500 index options. Our FMLS model is designed specifically to capture this feature of stock index options. As such, the model is not readily applicable to currency options. First, to generate finite price moments, we need to set the skewness of the $\alpha$-stable distribution to its negative maximum: $\beta=-1$. A symmetric $\alpha$-stable motion would generate infinite price moments. Hence, the model is incapable of capturing a symmetric smile. Second, under the FMLS model, the tail behavior does not vary with the conditional time horizon. While this feature is dearly needed for the S\&P 500 index options, it is in conflict with the stylized evidence on currency options. Indeed, traditional jump-diffusion models with stochastic volatility work much better for the currency options along the maturity dimension. The inapplicability of the FMLS model to currency options highlights the stark difference between the currency and stock index options markets. An intriguing avenue for future research is to investigate why the two asset markets exhibit such different behaviors.

\section{Asymptotic Behavior of the Implied Volatility Smirk}

Due to data constraints, our tests on the maturity pattern of the implied volatility smirk have been focused on maturities of less than two years. Therefore, the question of whether the smirk will flatten out eventually is not answered by the tests. Correspondingly, it remains an open question as whether the risk-neutral distribution for the index return converges to normality at very long maturities.

Complicating this question, the correspondence between the slope of the volatility smirk and the skewness of the risk-neutral distribution is at best an approximation. This correspondence gets progressively weaker as maturity increases. In particular, we have the following negative result: 
Proposition 2 To exclude arbitrage opportunities, the implied volatility smirk must flatten out asymptotically as maturity approaches infinity, as long as the level of the implied volatility remains finite.

Refer to Appendix A for a proof based on no-arbitrage arguments. It says that even if the return distribution does not converge to normality asymptotically, as in the case of our FMLS model, the volatility smirk will flatten out eventually, simply as a result of the definition of the implied volatility. A sufficient condition for this to hold is that the implied volatility does not explode as maturity increases. ${ }^{19}$ Our FMLS model satisfies this condition.

Insert Figure 5 About Here.

To compare the asymptotic behavior of implied volatility under the four models calibrated in Section III, we again adopt the mean parameter estimates in Table II and compute the implied volatilities at different maturities. In the left panel of Figure 5, we plot the term structure of the implied volatility over a maturity range of 25 years. For all four models, the at-the-money implied volatility increases with maturity. Furthermore, all four models seem to imply the existence of a finite implied volatility at long maturities, under the mean parameter estimates in Table II. The left panel depicts the term structure of the smirk slope around the money. The smirk slope implied by the VG and the MJD model flattens out very quickly, as expected from the fast convergence to normality for returns. Under the MJD-SV model, convergence to normality and hence flattening of the smirk is much slower. Under the FMLS model, the smirk slope flattens slowly as predicted by Proposition 2, even though the underlying return distribution never converges to normality.

\section{Summary and Future Research}

We document a particular maturity pattern of the implied volatility smirk in S\&P 500 index options. More specifically we find that the downward sloping smirk does not flatten out as maturity increases, 
at least within a moderate maturity range. To capture this invariance, we propose the finite moment logstable process (FMLS) for index levels. In the model, the log return has an $\alpha$-stable distribution and hence evades the wide net thrown by the assumptions of the central limit theorem. As a result, the return distribution does not converge to normal as maturity increases. We further restrict the $\alpha$ stable distribution to have a fat left tail, but a thin right tail. The asymmetry in the distribution not only generates the implied volatility smirk observed in the index options market, but also guarantees the existence of a martingale measure and finite option prices. Empirical calibration illustrates that the FMLS model provides a parsimonious way of modelling how implied volatilities vary in both the moneyness and maturity dimensions.

Future empirical research should look into documenting the maturity pattern for other indices and for individual stocks. Theoretical research should also be conducted into extensions of the FMLS model which are capable of capturing the finer details of price behavior in various options market. Our preliminary investigations suggest that stochastic time change (see Carr and $\mathrm{Wu}(2000)$ ) is a powerful tool which can profitably be used to capture such refinements. 


\section{Appendix A. Asymptotic Behavior of the Implied Volatility Smile}

This appendix proves that no arbitrage forces all finite implied volatility (IV) smirks or smiles which are asymptotically finite to also be asymptotically flat. This restriction is implicit in Hodges (1996), who presents an extensive list of arbitrage restrictions on the implied volatility surface. Another effort in this direction is Gatheral, Matytsin, and Youssfi (2000).

We assume frictionless markets and no arbitrage. We make no structural assumptions on interest rates and dividends, but we do assume that bond and forward prices are observable at all maturities. As a result, implied bond and dividend yields are observable at any maturity and we let $r_{t}^{T}$ and $q_{t}^{T}$ respectively denote these continuously compounded bond and dividend yields. For economy of notation, we will use $r$ and $q$ where the time and the term are understood. We assume that these term structures are bounded as $T \uparrow \infty$. Let $S_{t}$ and $C_{t}(K, \tau)$ respectively denote the time $t$ market prices of a stock and a European call on that stock with strike $K$ and maturity $\tau=T-t$. Assuming that these prices are arbitrage-free, the implied volatility surface at time $t$ is defined by,

$$
I V_{t}(K, \tau) \equiv B S C^{-1}\left(S_{t}, t ; K, \tau ; r, q, C_{t}(K, \tau)\right), \quad K>0, \tau>0,
$$

where $B S C^{-1}$ is the inverse of the Black-Scholes call formula in volatility. We assume that the implied volatility surface generated by (A1) is always finite. From (A1),

$$
C_{t}(K, \tau) \equiv B S C\left(S_{t}, t ; K, \tau ; r, q, I V_{t}(K, \tau)\right), \quad K>0, \tau>0 .
$$

We have used the call formula and call prices for definiteness, but under frictionless markets and no arbitrage, put prices must yield the same implied volatility surface as call prices:

$$
P_{t}(K, \tau) \equiv B S P\left(S_{t}, t ; K, \tau ; r, q, I V_{t}(K, \tau)\right), \quad K>0, \tau>0 .
$$

From Merton (1973), arbitrage-free call prices decrease with strike while put prices increase with strike. Assuming that the market call and put prices are differentiable in $K$, we have

$$
\begin{aligned}
\frac{\partial}{\partial K} C_{t}(K, \tau) & =\frac{\partial B S C}{\partial K}+\frac{\partial B S C}{\partial I V} \frac{\partial I V_{t}}{\partial K}(K, \tau) \leq 0 \\
\frac{\partial}{\partial K} P_{t}(K, \tau) & =\frac{\partial B S P}{\partial K}+\frac{\partial B S P}{\partial I V} \frac{\partial I V_{t}}{\partial K}(K, \tau) \geq 0 .
\end{aligned}
$$


Thus, the nonpositivity of the vertical call spread in (A2) bounds the implied volatility smirk slope from above while the nonnegativity of the vertical put spread in (A3) bounds the slope from below:

$$
-\frac{\frac{\partial B S P}{\partial K}}{\frac{\partial B S P}{\partial I V}} \leq \frac{\partial I V_{t}}{\partial K}(T, K) \leq-\frac{\frac{\partial B S C}{\partial K}}{\frac{\partial B S C}{\partial I V}} .
$$

By the Black-Scholes formula:

$$
\begin{array}{lll}
\frac{\partial B S P}{\partial K}=e^{-r \tau} N\left(-d_{2}\right), & & \frac{\partial B S P}{\partial I V}=K e^{-r \tau} \sqrt{\tau} N^{\prime}\left(d_{2}\right) ; \\
\frac{\partial B S C}{\partial K}=-e^{-r \tau} N\left(d_{2}\right), & \frac{\partial B S C}{\partial I V}=K e^{-r \tau} \sqrt{\tau} N^{\prime}\left(d_{2}\right),
\end{array}
$$

where $N(\cdot)$ is the cumulative density of a standard normal and $d_{2}$ is defined as:

$$
d_{2} \equiv \frac{\ln \left(S_{t} / K\right)+\left(r-q-\frac{1}{2} I V_{t}^{2}(K, \tau)\right) \tau}{I V_{t}(K, \tau) \sqrt{\tau}}
$$

Substituting (A5) into (A4) and rearranging, we have:

$$
-\frac{R\left(d_{2}\right)}{\sqrt{\tau}} \leq \frac{\partial I V_{t}}{\partial \ln K}(K, \tau) \leq \frac{R\left(-d_{2}\right)}{\sqrt{\tau}}
$$

where:

$$
R(d) \equiv \frac{1-N(d)}{N^{\prime}(d)}
$$

is known as Mill's Ratio. ${ }^{20}$ Loosely speaking, (A6) says that if the strike is increased by one percent, the implied volatility cannot decrease by more than Mill's ratio of $d_{2}$ divided by the square root of the time to maturity and cannot increase by more than Mill's ratio of $-d_{2}$ divided by the square root of the time to maturity.

The assumptions of finite term structures of interest rates and dividend yields and a finite implied volatility surface imply:

$$
\lim _{\tau \uparrow \infty}\left|d_{2}\right| \rightarrow \infty
$$

at the rate of $\sqrt{\tau}$. Using L'Hopital's rule on Mill's ratio:

$$
\lim _{\tau \uparrow \infty} R(d)=\lim _{d \rightarrow \pm \infty} R(d)=\lim _{d \rightarrow \pm \infty} \frac{1}{d}
$$

Hence, we have,

$$
-\lim _{\tau \uparrow \infty} \frac{R\left(d_{2}\right)}{\sqrt{\tau}}=-\lim _{\tau \uparrow \infty} O\left(-\frac{1}{\tau}\right) ; \quad \lim _{\tau \uparrow \infty} \frac{R\left(-d_{2}\right)}{\sqrt{\tau}} \lim _{\tau \uparrow \infty}=O\left(\frac{1}{\tau}\right)
$$


Letting $\tau \uparrow \infty$ in (A6), the substitution of (A7) in (A6) causes the implied volatility slope in strike to vanish asymptotically,

$$
\lim _{\tau \uparrow \infty} \frac{\partial I V_{t}}{\partial \ln K}(\tau, K)=0,
$$

at the rate of $1 / \tau$. Therefore, asymptotically, if implied volatility is graphed against the moneyness defined in this paper, $d \equiv \frac{\ln (K / F)}{\sqrt{\tau}}$, the smirk slope flattens out at the rate of $1 / \sqrt{\tau}$. 


\section{References}

Aï-Sahalia, Yacine, and Andrew Lo, 1998, Nonparametric estimation of state-price densities implicit in financial asset prices, Journal of Finance 53, 499-547.

Akaike, H., 1973, Information theory and an extension to the likelihood ratio principle, in B. N. Petrov, and F. Csaki, eds.: Proceedings of the Second International Symposium of Information Theory (Akademiai Kiado, Budapest ).

Backus, David, Silverio Foresi, and Liuren Wu, 1997, Accounting for biases in Black-Scholes, Working paper, New York University.

Bakshi, Gurdip, Charles Cao, and Zhiwu Chen, 1997, Empirical performance of alternative option pricing models, Journal of Finance 52, 2003-2049.

Bates, David, 1996, Jumps and stochastic volatility: Exchange rate processes implicit in Deutsche Mark options, Review of Financial Studies 9, 69-107.

Bates, David, 2000, Post-'87 crash fears in the s\&p 500 futures option market, Journal of Econometrics 94, $181-238$.

Black, Fisher, and Myron Scholes, 1973, The pricing of options and corporate liabilities, Journal of Political Economy 81, 637-654.

Carr, Peter, and Dilip B. Madan, 1999, Option valuation using the fast fourier transform, Journal of Computational Finance 2, 61-73.

Carr, Peter P., Hélyette Geman, Dilip B. Madan, and Marc Yor, 2000, The fine structure of asset returns: An empirical investigation, Journal of Business forthcoming.

Carr, Peter P., and Liuren Wu, 2000, Time-changed Lévy processes and option pricing, Working paper, Fordham University.

Carr, Peter P., and Liuren Wu, 2001, A simple robust test for the presence of jumps in asset prices, Working paper, Fordham University.

Das, Sanjiv Ranjan, and Rangarajan Sundaram, 1999, Of smiles and smirks: A term structure perspective, Journal of Financial and Qunatitative Analysis 34, 211-240.

Ding, Zhuanxin, Robert F. Engle, and Clive W. J. Granger, 1993, A long memory property of stock returns and a new model, Journal of Empirical Finance 1, 83-106.

Ding, Zhuanxin, and Clive W. J. Granger, 1996, Modeling volatility persistence of speculative returns: A new approach, Journal of Econometrics 73, 185-215. 
Fama, Eugene F., 1965, The behavior of stock market prices, Journal of Business 38, 34-105.

Gatheral, Jim, Andrew Matytsin, and Chrif Youssfi, 2000, The rational shape of the volatility surface, presentation, Merrill Lynch.

Heston, Stephen, 1993a, Closed-form solution for options with stochastic volatility, with application to bond and currency options, Review of Financial Studies 6, 327-343.

Heston, Stephen, 1993b, Invisible parameters in option prices, Journal of Finance 48, 933-947.

Hodges, Hrady M., 1996, Arbitrage bounds of the implied volatility strike and term structures of European-style options, Journal of Derivatives 3, 23-32.

Hurst, S. H., E. Platen, and Svetlozar T. Rachev, 1999, Option pricing for a logstable asset price model, Mathematical and Computer Modeling 29, 105-119.

Jackwerth, Jens Carsten, and Mark Rubinstein, 1996, Recovering probability distributions from contemporary security prices, Journal of Finance 51, 347-369.

Janicki, Aleksander, and Aleksander Weron, 1994, Simulation and Chaotic Behavior of $\alpha$-Stable Stochastic Processes. (Marcel Dekker New York).

Janicki, Aleksander W., Ivilina Popova, Peter H. Ritchken, and W.A. Woyczynski, 1997, Option pricing bounds in an $\alpha$-stable security market, Communication in Statistics - Stochastic Models 13, 817-839.

Konikov, Mikhail, and Dilip B. Madan, 2000, Pricing options of all strikes and maturities using a generalization of the VG model, Working paper, University of Maryland.

Leippold, Markus, and Liuren Wu, 2001, Asset pricing under the quadratic class, Journal of Financial and Quantitative Analysis forthcoming.

Lewis, Alan L., 2001, A simple option formula for general jump-diffusion and other exponential lévy processes, manuscript, Envision Financial Systems and OptionCity.net Newport Beach, California, USA.

Madan, Dilip B., Peter P. Carr, and Eric Chang, 1998, The variance gamma process and option pricing, European Financial Review 2, 79-105.

Mandelbrot, Benoit B., 1963, The variation of certain speculative prices, Journal of Business 36, 394-419.

McCulloch, J. Huston, 1987, Foreign exchange option pricing with log-stable uncertainty, in S. J. Khoury, and A. Ghosh, eds.: Recent Developments in International Banking and Finance (Lexington Books, Lexington, MA ).

McCulloch, J. Huston, 1996, Financial applications of stable distributions, in G. S. Maddala, and C. R. Rao, eds.: Statistical Methods in Finance (Elsevier Science, Amsterdam ). 
Merton, Robert C., 1973, Theory of rational option pricing, Bell Journal of Economics and Management Science 4, 141-183.

Merton, Robert C., 1976, Option pricing when underlying stock returns are discontinuous, Journal of Financial Economics 3, 125-144.

Patel, J., and C. Read, 1996, Handbook of the Normal Distribution. (Marcel Dekker New York).

Popova, Ivilina, and Peter Ritchken, 1998, On bounding option prices in paretian stable markets, Journal of Derivatives Summer, 32-43.

Rubinstein, Mark, 1994, Implied binomial trees, Journal of Finance 49, 771-818.

Samorodnitsky, Gennady, and Murad S. Taqqu, 1994, Stable Non-Gaussian Random Processes: Stochastic Models with Infinite Variance. (Chapman \& Hall New York).

Schwartz, G., 1978, Estimating the dimension of a model, Annals of Statistics 6, 461-464.

Simonoff, Jeffrey S., 1996, Smoothing Methods in Statistics. (Springer-Verlag New York).

Vuong, Quang H., 1989, Likelihood ratio tests for model selection and non-nested hypotheses, Econometrica 57, 307-333.

Zolotarev, Vladimir M., 1986, One-Dimensional Stable Distributions. (American Mathematical Society Providence, Rhode Island). 


\section{Notes}

${ }^{1}$ See, for example, Aï-Sahalia and Lo (1998), Jackwerth and Rubinstein (1996), and Rubinstein (1994) for empirical documentation of this phenomenon in S\&P 500 index options.

${ }^{2}$ Henceforth, our use of the term "implied volatility" refers only to those derived from prices of S\&P500 options, unless otherwise noted.

${ }^{3}$ See, for example, Backus, Foresi, and Wu (1997) and Das and Sundaram (1999) on the convergence to normality under stochastic volatilities.

${ }^{4}$ Note that $d$ is also the average of $-d_{1}$ and $-d_{2}$ appearing in the standard Black-Scholes put formula.

${ }^{5}$ Refer to Simonoff (1996) for a textbook treatment on the choice of kernels and bandwidths.

${ }^{6}$ We recognize that this linear relation cannot be maintained asymptotically.

${ }^{7}$ Out of the 290 business days, one day is particularly inactive and is deleted from the sample.

${ }^{8}$ We thank Takaki Hayashi for proposing this test.

${ }^{9}$ Refer to Samorodnitsky and Taqqu (1994) and Janicki and Weron (1994) for details on the process.

${ }^{10}$ For example, Merton (1976) conjectures that a symmetric $\alpha$-stable distribution specification for stock price would make a 5-minute call option worth 100 percent of its underlying stock. Merton (1976) further conjectures that this specification for the market portfolio might also require the equilibrium interest rate to be infinite.

${ }^{11}$ Recently, the FFT method of Carr and Madan (1999) has been extended to price any Europeanstyle options under Lévy processes by Lewis (2001) and under time-changed Lévy processes by Carr and $\mathrm{Wu}(2000)$. It has also been applied to price interest rate options by Leippold and $\mathrm{Wu}(2001)$.

${ }^{12}$ The slope of the implied volatility smirk is estimated by a simple linear regression. The moneyness is confined to the approximately linear range of $[-2,0]$ for the regression. 
${ }^{13}$ The vector $\Theta$ also includes the current level of the instantaneous variance $v_{t}$ in the MJD-SV model.

${ }^{14}$ Such a discrepancy arises when put-call parity is violated and the two option quotes do not yield the same implied volatility.

${ }^{15}$ We also experimented with cubic splines. The results are almost identical.

${ }^{16}$ The state variable $v_{t}$ is essentially treated as another free parameter in our estimation procedure.

${ }^{17}$ Note that while the daily average of the mse for the MJD-SV model is smaller than that for the FMLS model, the average of the unadjusted likelihood ratio test $\hat{M}_{0}$ is actually positive, albeit insignificant. The difference comes from the different space in averaging: the likelihood ratio test averages on the log of mse instead of mse itself.

${ }^{18}$ Under the MJD model, the annual variance of the log return is given by

$$
\kappa_{2}=\left[\sigma^{2}+\alpha\left(\omega^{2}+\eta^{2}\right)\right]
$$

The fraction of variation explained by the diffusion component is $\sigma^{2} / \kappa_{2}$.

${ }^{19}$ The condition will be violated, for example, when the stochastic volatility process is non-stationary.

${ }^{20}$ See Patel and Read (1996), pages 55-66, for various approximations. 


\section{List of Figures}

1 Volatility Smirk in S\&P 500 Index Options. The implied volatility surface in the left panel is obtained via nonparametric smoothing of daily closing implied volatility quotes (mid point of bid and ask) on S\&P 500 index options from April 4th, 1999 to May 31st, 2000 (62,950 observations). Independent Gaussian kernels are used with default bandwidth choices. Moneyness, $d$, is defined as $d=\ln (K / F) / \sigma \sqrt{\tau}$, where $\sigma=27.4 \%$ is the average of all implied volatility quotes. Lines in the right panel are two-dimensional cuts of the surface at maturities of 1 month (solid line), 6 months (dashed line), 12 months (dash-dotted line), and 15 months (dotted line). . . . .

2 Maturity Effect on Volatility Smirk. Lines in the left panel are the term structure of the estimated smirk slopes at each day. The solid line in the right panel depicts a linear regression fit to the stacked data. The smirk slope at each date and maturity is estimated by regressing the implied volatility $I V$ on the moneyness $d$ within the approximately linear range of $d \in[0,2]$. .

3 Volatility Smirk Under FMLS Model. Implied volatilities are computed from the FMLS model with $\sigma=0.14, r=7.33 \%$ and $q=1.17 \%$. In the left panel, the maturity is fixed at one month while the tail index varies from 1.2 (solid line) to 1.5 (dashed line), to 1.8 (dash-dotted line), and to 2.0 (dotted line). In the right panel, the tail index is fixed at $\alpha=1.5$ while the maturity of the option varies from one month (solid line), to six month (dashed line), and to one year (dash-dotted line) . . . . . . . . . . . . . . . . . . . . 36

4 The Maturity Pattern of Volatility Smirk Implied by Different Models Implied volatilities are computed from the FMLS model (top left), MJD-SV model (top right), VG model (bottom left) and MJD model (bottom right). Model parameters are chosen to match the mean estimates reported in Table II. We further set interest rate $r=7.33 \%$ and dividend yields $q=1.17 \%$. Maturity $\tau$ equals 1 month (solid line), 6 months (dashed line), and 12 months (dash-dotted line). 37

$5 \quad$ Asymptotic Behavior of the Implied Volatility Smirk. $\ldots \ldots \ldots \ldots$

\section{List of Tables}

I Regression Tests on the Maturity Pattern of the Implied Volatility Smirk . . . . . . . . . . . 39

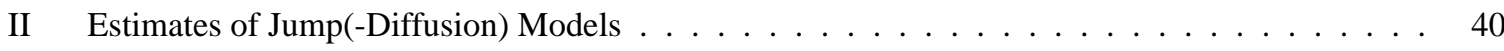



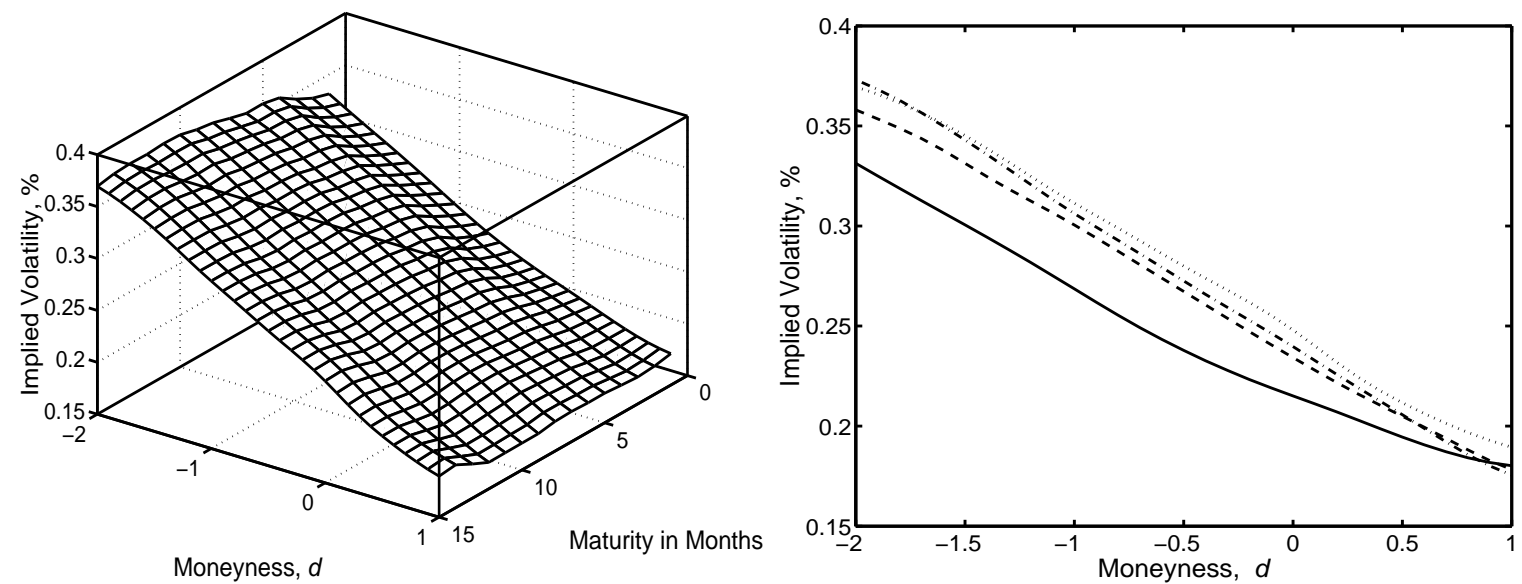

Figure 1. Volatility Smirk in S\&P 500 Index Options. The implied volatility surface in the left panel is obtained via nonparametric smoothing of daily closing implied volatility quotes (mid point of bid and ask) on S\&P 500 index options from April 4th, 1999 to May 31st, 2000 (62,950 observations). Independent Gaussian kernels are used with default bandwidth choices. Moneyness, $d$, is defined as $d=\ln (K / F) / \sigma \sqrt{\tau}$, where $\sigma=27.4 \%$ is the average of all implied volatility quotes. Lines in the right panel are two-dimensional cuts of the surface at maturities of 1 month (solid line), 6 months (dashed line), 12 months (dash-dotted line), and 15 months (dotted line).
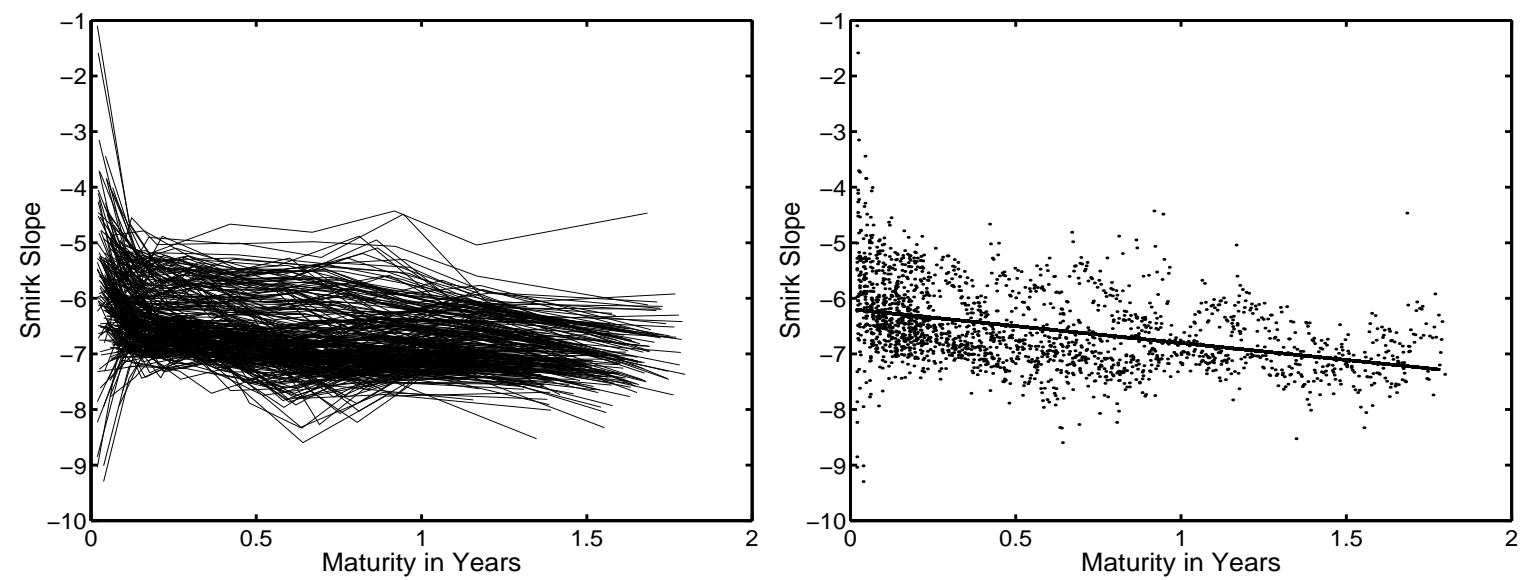

Figure 2. Maturity Effect on Volatility Smirk. Lines in the left panel are the term structure of the estimated smirk slopes at each day. The solid line in the right panel depicts a linear regression fit to the stacked data. The smirk slope at each date and maturity is estimated by regressing the implied volatility $I V$ on the moneyness $d$ within the approximately linear range of $d \in[0,2]$. 

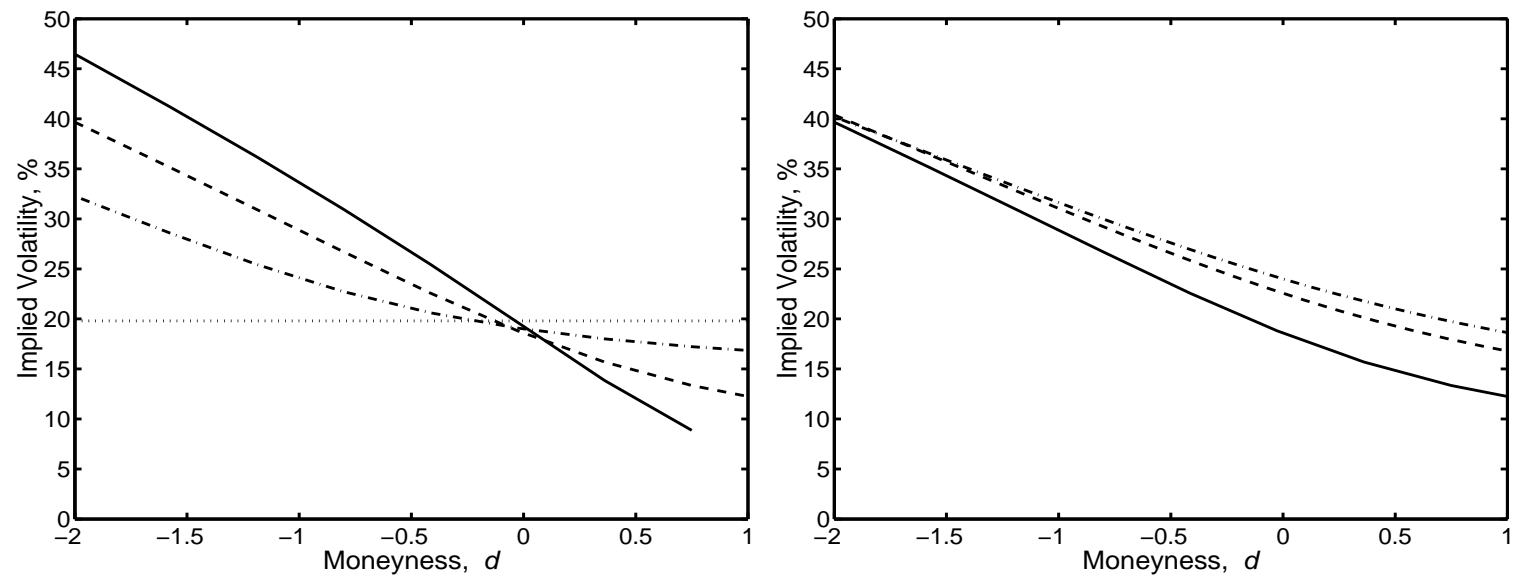

Figure 3. Volatility Smirk Under FMLS Model. Implied volatilities are computed from the FMLS model with $\sigma=0.14, r=7.33 \%$ and $q=1.17 \%$. In the left panel, the maturity is fixed at one month while the tail index varies from 1.2 (solid line) to 1.5 (dashed line), to 1.8 (dash-dotted line), and to 2.0 (dotted line). In the right panel, the tail index is fixed at $\alpha=1.5$ while the maturity of the option varies from one month (solid line), to six month (dashed line), and to one year (dash-dotted line). 

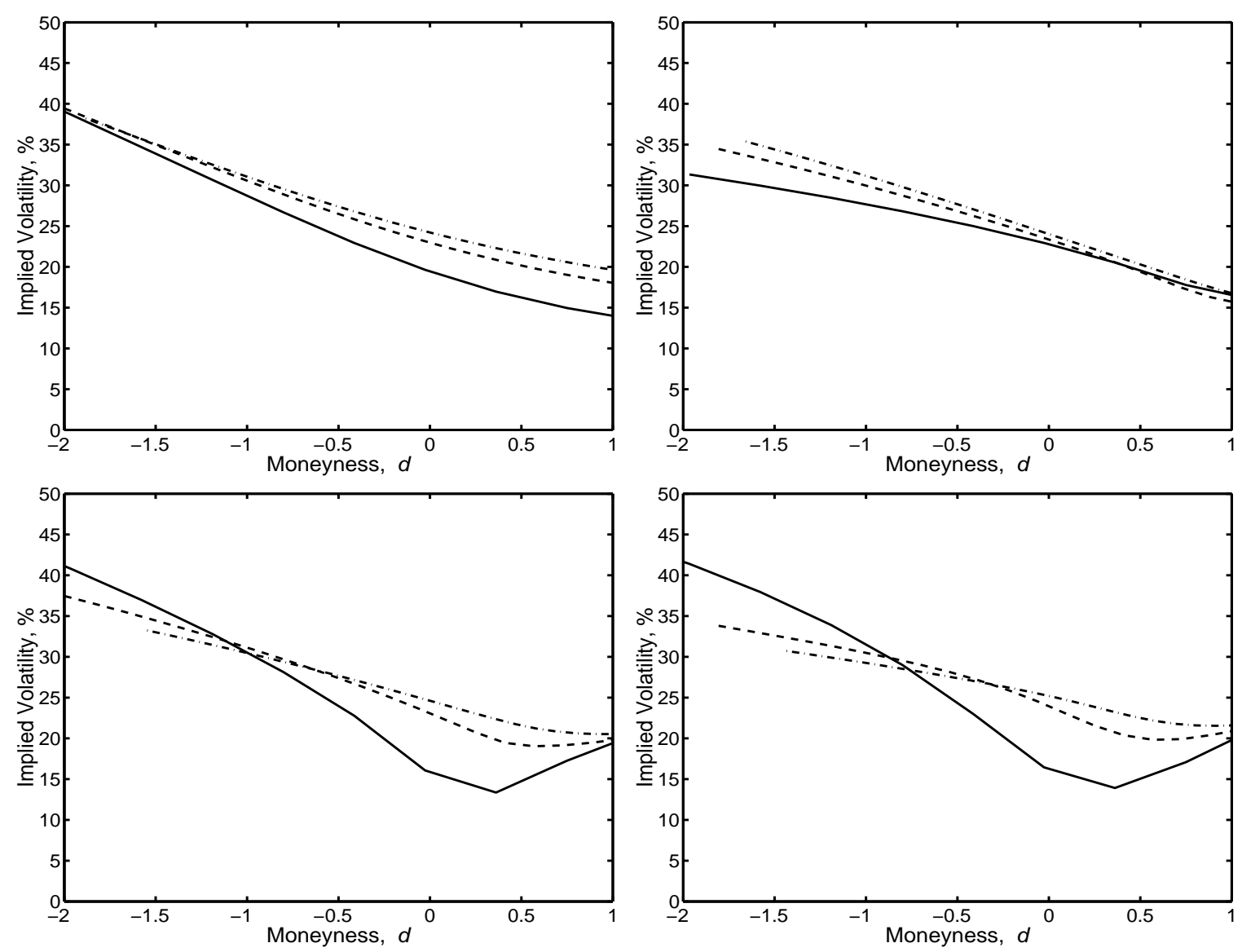

Figure 4. The Maturity Pattern of Volatility Smirk Implied by Different Models Implied volatilities are computed from the FMLS model (top left), MJD-SV model (top right), VG model (bottom left) and MJD model (bottom right). Model parameters are chosen to match the mean estimates reported in Table II. We further set interest rate $r=7.33 \%$ and dividend yields $q=1.17 \%$. Maturity $\tau$ equals 1 month (solid line), 6 months (dashed line), and 12 months (dash-dotted line). 

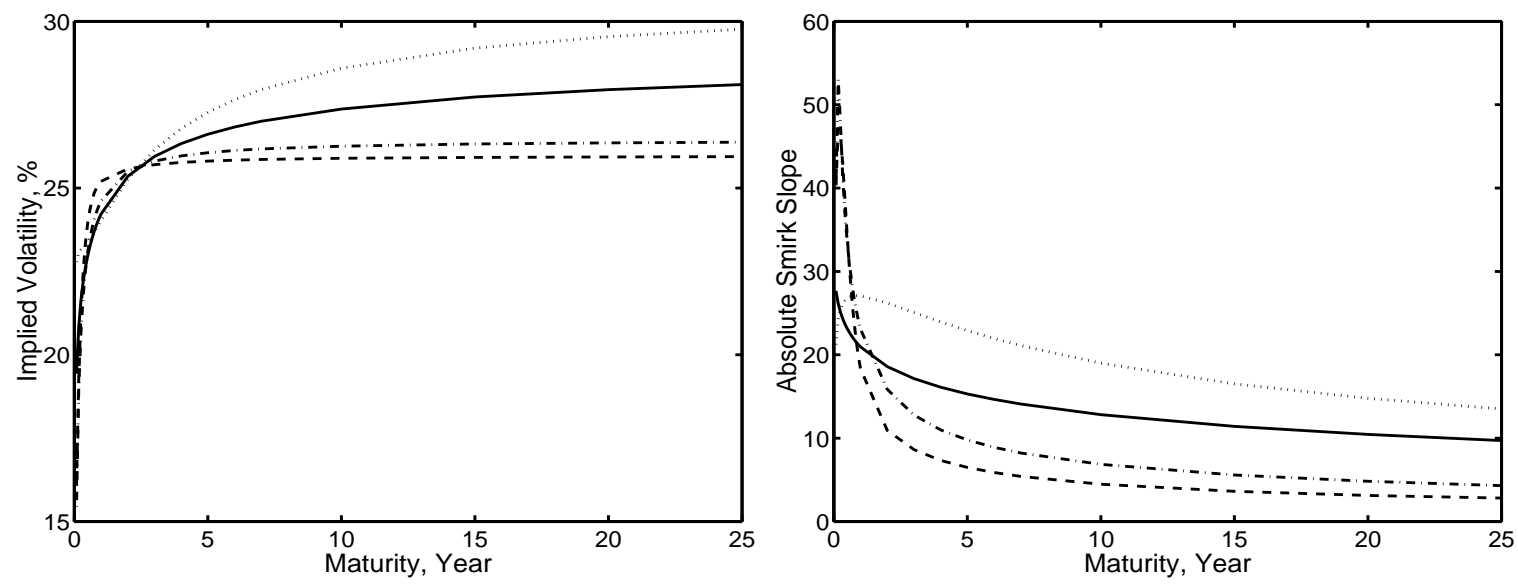

Figure 5. Asymptotic Behavior of the Implied Volatility Smirk.

The left panel plots the term structure of the at-the-money implied volatility and the right panel plots the term structure of the absolute value of the slope of the near-the-money volatility smirk, defined as $\mid$ slope $\mid=\frac{\left|I V_{1}-I V_{2}\right|}{\left|d_{1}-d_{2}\right|}$ where $d_{1}=<0$ and $d_{2}=0$. The four lines are implied by the four models: FMLS (solid line), VG (dashed line), MJD (dash-dotted line), and MJD-SV (dotted line), with mean parameter estimates reported in Table II. We further set $r=q=0$. 
Table I

Regression Tests on the Maturity Pattern of the Implied Volatility Smirk

Panel A reports the estimates of the following stacked regression,

$$
b_{j}=c_{0}+c \tau_{j}+\varepsilon
$$

where $b_{j}$ is the smirk slope estimate at each maturity and date from the following regression,

$$
I V_{j}=a+b_{j} d_{j}+e_{j}
$$

and $\tau_{j}$ denotes the corresponding maturity. The moneyness is defined as $d=\ln (K / F) / \sigma \sqrt{\tau}$ and is restricted to $[0,2]$ for the regression. Panel B reports the estimates of an alternative stacked one-step regression:

$$
I V=c_{0}+c_{1} d+c_{2} \tau+c_{3}(\tau d)+e .
$$

All regressions are based on 289 active business days of implied volatility quotes on S\&P 500 index options across different strikes and maturities, from April 4th, 1999 to May 31st, 2000.

A. Stacked two-step regression

\begin{tabular}{lrr}
\hline & $c_{0}$ & $c$ \\
Estimates & -6.20 & -266.11 \\
$t$-statistics & -0.61 & -19.32 \\
& & \\
\hline
\end{tabular}

B. Stacked one-step regression

\begin{tabular}{lrrrr}
\hline & $c_{0}$ & $c_{1}$ & $c_{2}$ & $c_{3}$ \\
Estimates & 21.48 & -6.14 & 2.42 & -0.79 \\
$t$-statistics & 151.14 & -164.20 & 51.44 & -15.00 \\
& & & & \\
\hline
\end{tabular}


Table II

\section{Estimates of Jump(-Diffusion) Models}

Model parameters are estimated at each day by minimizing the sum of squared pricing error between the observed and model-implied out-of-the-money option prices (call prices when $K>F$, puts when $K \leq F$ ) on S\&P 500 index options across all available strikes and maturities. Entries report the sample average of the daily estimates of the model parameters, followed in parentheses by its standard deviation. The sample contains 289 active business days, from April 4th, 1999 to May 31st, 2000. Also reported are the sample averages (and standard deviations in parentheses) of the daily estimates on the mean squared pricing error (mse) and the three likelihood ratio test statistics $\hat{M}_{j}(j=0,1,2)$ between the FMLS model and the other three models. All three tests are asymptotically normally distributed with zero mean and unit variance. A significantly positive statistic indicates the better performance of the FMLS model and a negative statistic indicates the better performance of the other model. The second column (FMLS) denotes the estimates for our finite moment log stable model. The third column (VG) reports the estimates for the variance-gamma model of Madan, Carr, and Chang (1998), the fourth column (MJ) denotes the Poisson jump model of Merton (1976), the last column (MJ-SV) denotes the MJ model augmented with a stochastic volatility process as in Heston (1993a).

\begin{tabular}{|c|c|c|c|c|}
\hline Parameters & FMLS & VG & MJD & MJD-SV \\
\hline \multirow[t]{2}{*}{$\alpha$} & 1.5597 & 0.7681 & 1.8145 & 1.8257 \\
\hline & $(0.0466)$ & $(0.0679$ & $(0.0342)$ & $(0.0991)$ \\
\hline \multirow[t]{2}{*}{$\sigma$} & 0.1486 & 0.2295 & 0.0638 & - \\
\hline & $(0.0110)$ & $(0.0202)$ & $(0.0185)$ & - \\
\hline \multirow[t]{2}{*}{$\omega$} & - & -0.2013 & -0.1045 & -0.0528 \\
\hline & - & $(0.0205)$ & $(0.0132)$ & $(0.0232)$ \\
\hline \multirow[t]{2}{*}{$\eta$} & - & - & 0.1671 & 0.0663 \\
\hline & - & - & $(0.0177)$ & $(0.0026)$ \\
\hline \multirow[t]{2}{*}{$\theta$} & - & - & - & 0.1184 \\
\hline & - & - & - & $(0.0381)$ \\
\hline \multirow[t]{2}{*}{$\kappa$} & - & - & - & 0.8480 \\
\hline & - & - & - & $(0.1564)$ \\
\hline \multirow[t]{2}{*}{$\sigma_{v}$} & - & - & - & 0.6623 \\
\hline & - & - & - & $(0.0924)$ \\
\hline \multirow[t]{2}{*}{$\rho$} & - & - & - & -0.8884 \\
\hline & - & - & - & $(0.0464)$ \\
\hline \multirow[t]{2}{*}{$\operatorname{mse}\left(\times 10^{5}\right)$} & 0.3630 & 0.8042 & 1.4473 & 0.1452 \\
\hline & $(0.1297)$ & $(0.1841)$ & $(0.3665)$ & $(0.0770)$ \\
\hline \multirow[t]{2}{*}{$\hat{M}_{0}$} & - & 2.6790 & 3.8127 & 1.0960 \\
\hline & - & $(2.0692)$ & 3.2501 & $(2.4399)$ \\
\hline \multirow[t]{2}{*}{$\hat{M}_{1}$} & - & 3.6790 & 5.8127 & 7.0960 \\
\hline & - & $(2.0692)$ & 3.2501 & $(2.4399)$ \\
\hline \multirow[t]{2}{*}{$\hat{M}_{2}$} & - & 5.0727 & 8.6002 & 15.4585 \\
\hline & - & $(2.0692)$ & $(3.2501)$ & (2.4399) \\
\hline
\end{tabular}

